\title{
Loss of ALCAM expression is linked to adverse phenotype and poor prognosis in breast cancer: A TMA-based immunohistochemical study on 2,197 breast cancer patients
}

\author{
EIKE BURANDT $^{1}$, TANAZ BARI NOUBAR ${ }^{1}$, ANNETTE LEBEAU $^{1}$, SARAH MINNER $^{1}$, \\ CHRISTOPH BURDELSKI ${ }^{1}$, FRITZ JÄNICKE ${ }^{2}$, VOLLKMAR MÜLLER ${ }^{2}$, LUIGI TERRACCIANO ${ }^{3}$, \\ RONALD SIMON $^{1}$, GUIDO SAUTER ${ }^{1}$, WALDEMAR WILCZAK ${ }^{1}$ and PATRICK LEBOK ${ }^{1}$ \\ Departments of ${ }^{1}$ Pathology and ${ }^{2}$ Gynecology, University Medical Center Hamburg-Eppendorf, \\ Hamburg, Germany; ${ }^{3}$ Department of Pathology, University Hospital, Basel, Switzerland
}

Received July 30, 2014; Accepted August 25, 2014

DOI: $10.3892 /$ or.2014.3523

\begin{abstract}
Activated leukocyte cell adhesion molecule (ALCAM) is a membranous cell adhesion protein that is often expressed in breast cancer. Data on the prognostic impact of ALCAM expression is highly controversial in this cancer. To evaluate the clinical impact of ALCAM expression in a sufficiently large patient cohort, we utilized a tissue microarray (TMA) containing more than 2,100 primary breast cancers with clinical follow-up data by immunohistochemistry. TMA spots containing normal breast epithelium showed moderate to strong membranous ALCAM staining. ALCAM staining was strong in $66.2 \%$, moderate in $10.9 \%$, weak in $11.1 \%$ and absent in $11.8 \%$ of 1,778 (80.9\%) interpretable breast cancer tissue spots. Decreased ALCAM expression was significantly associated with advanced tumor size $(\mathrm{p}=0.0017)$, unfavorable tumor grade $(\mathrm{p}<0.0001)$, negative ER and PR status $(\mathrm{p}<0.0001$ each) as well as high Ki67 labeling index $(\mathrm{p}<0.0001)$. Cancers with ACLAM expression loss had a significantly poorer overall $(\mathrm{p}<0.0001)$ and disease-specific survival $(\mathrm{p}=0.0088)$. This association also held true in the subset of nodal positive cancers $(\mathrm{p}<0.0001)$. In conclusion, these data demonstrate that ALCAM is generally expressed in normal and cancerous breast epithelium and that a marked reduction of ALCAM expression characterizes a subset of breast cancer patients with adverse tumor characteristics and unfavorable clinical outcome.
\end{abstract}

Correspondence to: Dr Eike Burandt, Department of Pathology, University Medical Center Hamburg-Eppendorf, Martinistrasse 52, D-20246 Hamburg, Germany

E-mail: e.burandt@uke.de

Key words: breast cancer, tissue microarray, immunohistochemistry, ALCAM, prognosis

\section{Introduction}

Activated leukocyte cell adhesion molecule (ALCAM), also designated CD166, is a member of the immunoglobulin superfamily. ALCAM is a cell adhesion molecule expressed by epithelial cells in several organs and is involved in embryogenesis, angiogenesis, hematopoiesis (1), immune response (2) and, of course, cell adhesion (3). Cell adhesion molecules can be involved in tumor cell-tumor cell adhesion, tumor cellendothelial cell adhesion and tumor cell-matrix adhesion, which are all essential during primary tumor formation or metastasis at different times.

The initial studies that identified a role for ALCAM in human malignancies were performed in melanoma $(4,5)$. Over the last decade, alterations in ALCAM expression have been described in several other malignancies including bladder (6), colorectal (7), esophageal squamous cell (8), pancreatic (9), oral squamous cell (10) and ovarian cancer (11), neuroblastoma (12), prostate (13) and breast cancer $(14,15)$. These data suggest that ALCAM expression is increased in some tumors and downregulated in others. An association between high ALCAM expression and unfavorable prognosis has been shown for colorectal (7), pancreatic (9) and esophageal squamous cell cancer (8), and neuroblastoma (12). As a membrane protein, ALCAM also represents a potential target for therapy, which has already been successfully targeted by human recombinant single-chain antibody in breast cancer cells (16).

For breast cancer, ALCAM expression data are contradictory. Some studies revealed an increased ALCAM expression to be associated with poor prognosis $(15,17)$ or lymph node metastasis and local recurrence (18), while others found a decreased ALCAM expression to be linked to a poorer clinical course $(14,19,20)$. One explanation for these conflicting data potentially lies in the relatively small number of breast cancer cases included in those studies, ranging from 56 to 347 $(14,15,17,21)$. The aim of the present study was to clarify the prevalence and prognostic role of ALCAM expression in breast cancer using a pre-existing large-scale tissue microarray (TMA) including more than 2,000 breast cancer samples. Our data demonstrate a strong link between reduced membranous 
ALCAM expression and adverse phenotype and poor prognosis in breast cancer.

\section{Materials and methods}

Breast cancer TMA. The pre-existing TMA used for the purpose of the present study has been described in detail previously (22). In brief, the TMA contained a total of 2,197 human breast cancer samples from paraffin-embedded tissue specimens fixed in $4 \%$ neutral buffered formalin. The median patient age was 62 years (range, 26-101). The use of the specimens and data for research purposes was approved by the local Ethics Committee of the University of Basel. Survival data were either obtained from the cancer registry of Basel or collected from the patient attending physicians. At the end of the present study, there were 2,194 patients with overall survival data (710 patients with, and 1,484 without event) and 943 with disease-specific data (204 patients with, and 739 without event) (Fig. 1). The mean follow-up time was 68 months (range, 1-176). Tumor size and nodal status were obtained from the primary pathology reports. All slides from the tumors were reviewed by specialized pathologists to define the histologic grade according to Elston and Ellis (23) and the tumor type according to the WHO classification (WHO 2012). Four micrometer sections of the TMA blocks were transferred to an adhesive-coated slide system (Instrumedics Inc., Hackensack, NJ, USA) for immunohistochemical analyses. Evaluation of ALCAM expression status was in accordance with the reporting recommendations for tumor marker prognostic studies (REMARK) guidelines (24).

Immunohistochemistry (IHC). Freshly cut TMA sections were stained on one day in a single experiment. High-temperature pretreatment of slides was carried out in an autoclave in citrate buffer, $\mathrm{pH} 7.8$ for $5 \mathrm{~min}$. Immunostaining for ALCAM was performed using a monoclonal antibody (clone: MOG/07, 1:450; Novocastra). The EnVision system (Dako) was used to visualize the immunostaining. Tissue with known positivity was used as positive control and tonsil lymphocytes were used as negative controls. All samples were evaluated by one of the authors (E.B.). The staining intensity (scored on a scale of 0 to $3+$ ) and the proportion of positive tumor cells was recorded for each tissue spot. Only membranous staining was evaluated since cytoplasmic staining, if present, was always linked with stronger membranous staining. Based on these values, a final IHC result was calculated according to the following criteria: negative, in case of no staining at all, and positive, subclassified as weak, moderate and strong (weak, 1+ staining intensity in $\leq 70 \%$ positive tumor cells or $2+$ staining intensity in $\leq 30 \%$ positive tumor cells; moderate, $1+$ staining intensity in $>70 \%$ tumor cells, $2+$ in $>30 \%$ but $\leq 70 \%$ positive tumor cells or $3+$ in $\leq 30 \%$ positive tumor cells; strong, $2+>70 \%$ or $3+>30 \%$ positive tumor cells). Data on the immunohistochemical expression of Ki67, ER and PR were available from previous studies $(22,25)$. In these studies, the Allred score was utilized for the evaluation of ER/PR staining. The percentage of Ki67-positive tumor cell nuclei was registered as Ki67 labeling index (LI).

Statistical analysis. Contingency table analysis and Chi-square test were used to study the relationship between IHC results

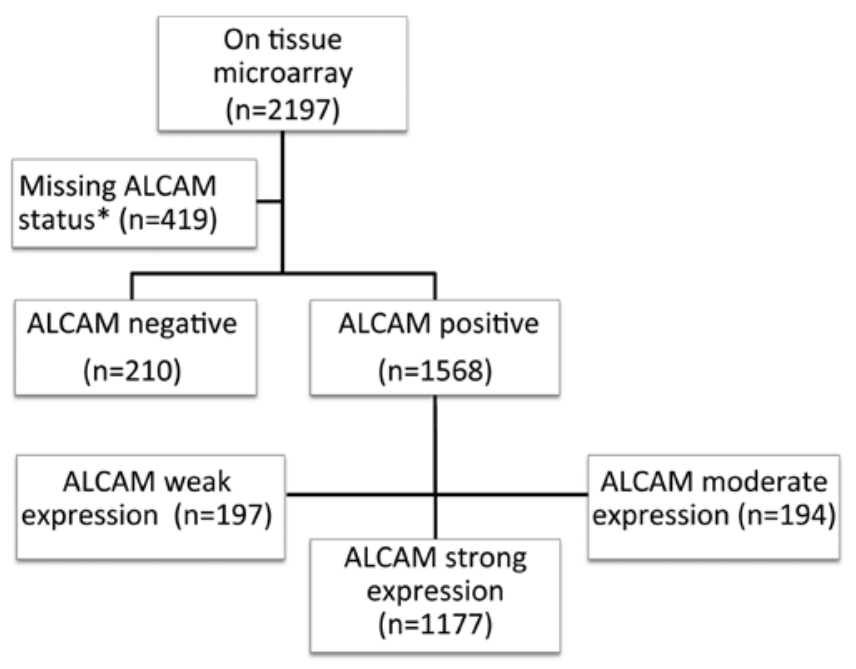

Figure 1. Schematic REMARK diagram representing the results for ALCAM immunohistochemistry analysis. "Missing ALCAM status was due to the absence of tissue on the TMA, lack of unequivocal tumor cells in the arrayed sample or by insufficient immunohistochemistry.

and clinicopathological variables. Kaplan-Meier plots were used to estimate disease-specific and overall survival and the statistical significance was determined by the log-rank test. Cox proportional hazard model with stepwise selection of the covariates was used to determine the parameters with greatest influence on patient survival. In exploratory analysis, $p$-values $<0.05$ were considered to indicate a statistically significant difference.

\section{Results}

ALCAM expression. ALCAM immunostaining was interpretable in $1,778(80.9 \%)$ of the 2,197 arrayed tissue spots on the TMA. Analysis failures were either due to the absence of tissue on the TMA or lack of unequivocal tumor cells in the arrayed sample. ALCAM immunostaining always showed strong membrane predominance in our tissues. Although varying degree of cytoplasmic staining was sometimes observed, this was always associated with a much higher staining level at the membranes. Membranous ALCAM staining was observed in $1,568(88.2 \%)$ of the informative cases. Staining was considered weak in $197(11.1 \%)$, moderate in 194 (10.9\%) and strong in $1,177(66.2 \%)$ tumors. ALCAM immunostaining was absent in $210(11.8 \%)$ cases. Representative images of ALCAM staining in breast cancers are shown in Fig. 2.

ALCAM expression and tumor phenotype. ALCAM staining levels were comparable (60-90\% with strong staining) in most different histological subtypes except from medullary cancers, which showed a significantly lower fraction of strongly ALCAM-positive tumors [36\%, as compared to $66 \%$ in ductal carcinomas/invasive cancer of no special type (NST); WHO 2012] $(p<0.0001)$. Reduced ALCAM staining was significantly associated with advanced tumor size $(\mathrm{p}=0.0017)$ and unfavorable tumor grade $(\mathrm{p}<0.0001)$. ALCAM staining levels were unrelated to nodal status $(\mathrm{p}=0.098)$. In ductal carcinomas (NST), the largest histologically defined subgroup, the asso- 

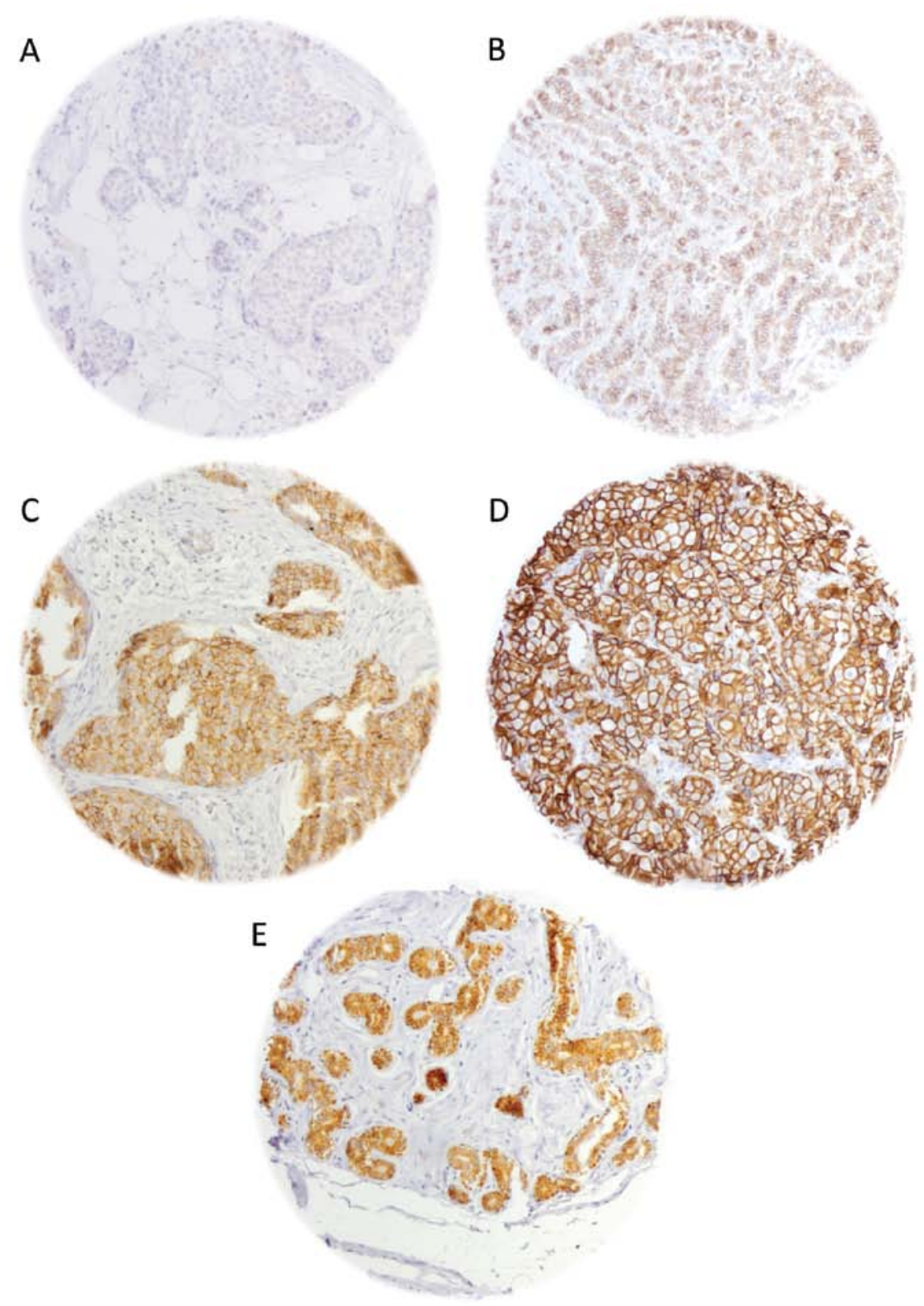

Figure 2. Images of ALCAM immunostaining. (A-D) Breast cancers with negative (A), weak (B), moderate (C) and strong (D) ALCAM expression; (E) normal breast epithelium showing moderate to strong ALCAM expression.

ciations with tumor phenotype were similar; loss of ALCAM expression was significantly linked to advanced tumor size $(p=0.0015)$ and higher tumor grade $(p<0.0001)$. No significant association was found between ALCAM staining levels and nodal status in the ductal (NST) subgroup ( $\mathrm{p}=0.045)$.

Cell proliferation was previously determined immunohistochemically by using the Ki67 LI (22). An inverse relationship was found between ALCAM staining and cell proliferation; the average Ki67 LI increased from $26.3 \%$ in 1,011 cancers with strong ALCAM staining to $33.1 \%$ in 181 tumors lacking detectable ALCAM expression ( $\mathrm{p}<0.0001$; Table I). Reduced ALCAM immunostaining was further linked to negative ER and PR status $(\mathrm{p}<0.0001$ each). All results are summarized in Table II.

ALCAM expression as prognostic factor for survival. Survival analysis revealed a highly significant relationship between
Table I. Correlation between ALCAM expression levels and Ki67 LI.

\begin{tabular}{lrcc}
\hline ALCAM score & $\mathrm{n}$ & Ki67 LI (mean) & P-value \\
\hline Negative & 181 & 33.1 & $<0.0001$ \\
Weak & 170 & 30.1 & \\
Moderate & 160 & 27.5 & \\
Strong & 1,011 & 26.3 & \\
\hline
\end{tabular}

LI, labeling index.

loss of ALCAM expression and poor overall $(\mathrm{p}<0.0001$; Fig. 3A) as well as disease-specific patient survival $(\mathrm{p}=0.0088$; Fig. 3B). This association also held true in the subset of nodal 
Table II. Pathological characteristics of the analyzed breast cancers included in the TMA in correlation with the ALCAM IHC results.

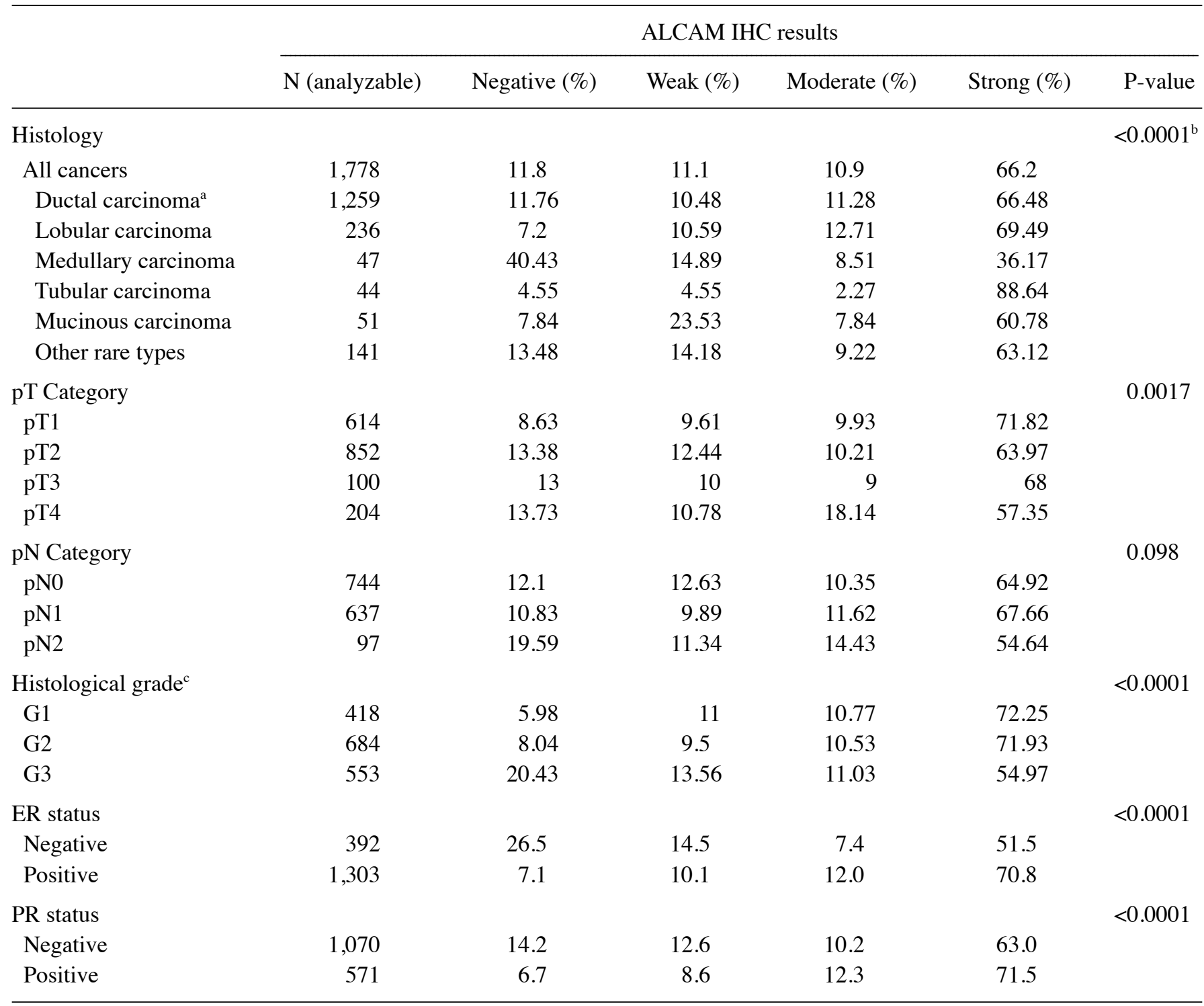

${ }^{a}$ Invasive carcinoma of no special type (NST; WHO 2012). ${ }^{b}$ Medullary vs. ductal carcinoma. ${ }^{\text {cE}}$ Elston and Ellis (23).

positive breast cancers ( $\mathrm{p}<0.0001$; Fig. 3C). An additional analysis in a subset of 202 breast cancer patients who had received tamoxifen monotherapy revealed no significant association between ALCAM expression and survival data $(\mathrm{p}=0.2447$; data not shown). Separate analyses were also performed for subgroups with alternative treatment information. In the subgroup of 63 breast cancer patients who were treated by chemotherapy alone, the relationship between ALCAM expression and outcome was retained $(p=0.0212$; data not shown). In the 69 patients with combined treatment of chemotherapy and tamoxifen, there was no significant impact of ALCAM expression on survival ( $\mathrm{p}=0.2486$; data not shown).

A multivariate analysis including tumor size, nodal status and histological grade did not suggest that ALCAM expression is an independent prognostic marker in our patient cohort (Table III).

\section{Discussion}

In the present study, more than 1,700 breast cancers were successfully analyzed for ALCAM expression. The results suggest that high ALCAM expression is a common feature of normal and cancerous breast epithelial cells and that its loss is linked to tumor progression, rapid cell proliferation and shortened survival.

A comparison with normal breast tissue revealed that ALCAM expression is high in normal breast epithelium but is reduced or lost in a fraction of cancers. This observation is in line with earlier data from King et al (14) and Kilic et al (26) describing that high levels of ALCAM expression are typically seen in normal breast epithelium. These findings suggest that high levels of ALCAM expression represent a physiological situation. ALCAM expression was found to be retained at high frequency and high levels in breast cancer in the present 
A

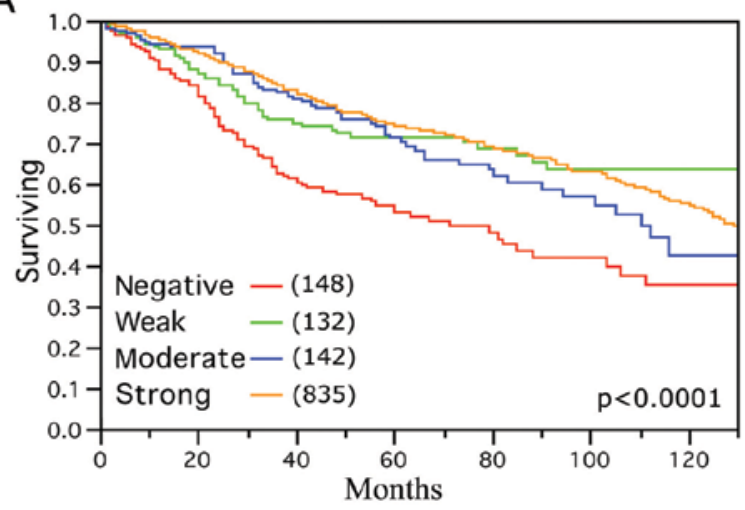

B

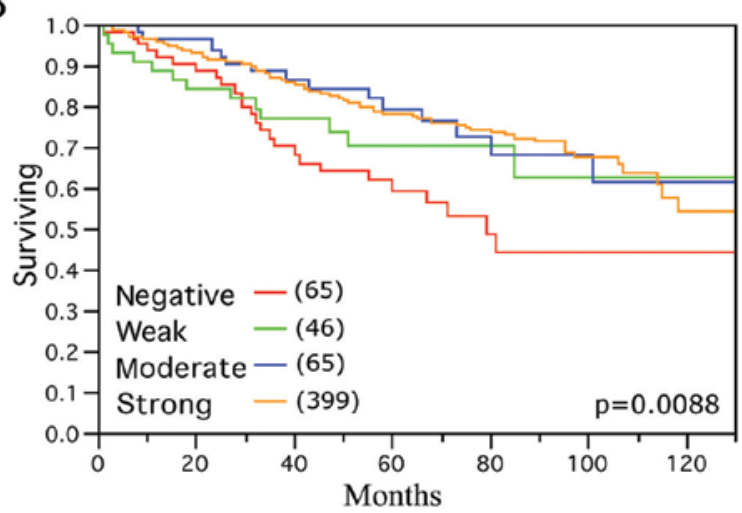

C

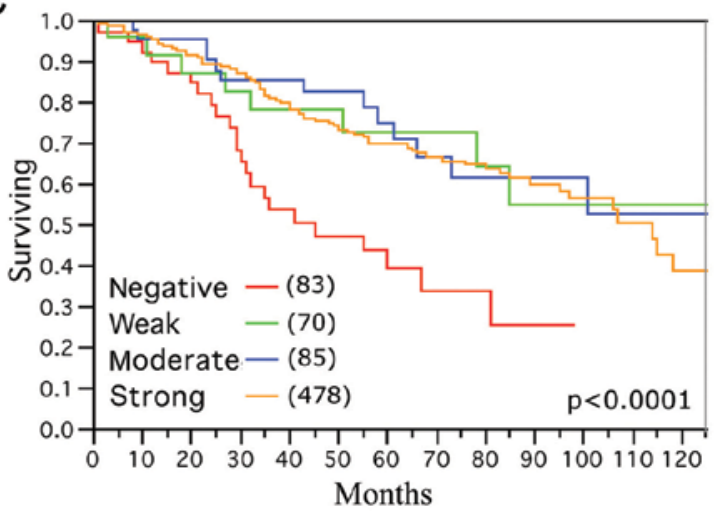

Figure 3. Kaplan-Meier survival curves showing the prognostic significance of ALCAM expression. (A) All cancers, overall survival; (B) all cancers, disease-specific survival; (C) subset of nodal positive cancers, overall survival.

study. A total of $88.2 \%$ of our cancers had detectable expression including $66.2 \%$ with 'strong' expression according to our scoring criteria. This high frequency is consistent with data from several earlier studies. Burkhardt et al (15), for example, found $75 \%$ ALCAM-expressing cancers among 160 analyzed cases. Ihnen et al (27) showed that 95\% of their 162 breast cancers were ALCAM-positive, while Hein et al (17) demonstrated 79\% ALCAM-expressing breast cancers in their cohort comprising 347 cases. Another study analyzing 84 cases found $96 \%$ of the tested breast cancers to be ALCAM-positive (28).

In the present study, a significant association between lost ALCAM expression and an adverse clinical course was observed. This finding is in line with several of the earlier
Table III. COX proportional hazard multivariate analysis (tumor specific).

\begin{tabular}{llc}
\hline & \multicolumn{2}{c}{ Disease-specific survival } \\
\cline { 2 - 3 } & HR $(95 \%$ CI $)$ & P-value \\
\hline ALCAM expression & & 0.3349 \\
Negative vs. weak & $1.4(1.0-2.1)$ & \\
Weak vs. moderate & $0.9(0.4-1.5)$ & \\
Moderate vs. strong & $0.9(0.5-1.3)$ & \\
Size (pT) & & 0.008 \\
pT1 vs. pT2 & $0.8(0.6-1.2)$ & \\
pT2 vs. pT3 & $1.0(0.7-1.4)$ & \\
pT3 vs. pT4 & $0.7(0.3-1.1)$ & \\
Nodal status & & \\
pN0 vs. pN1 & $0.3(0.2-0.4)$ & \\
pN1 vs. pN2 & $1.0(0.8-1.3)$ & \\
Grade & & \\
G1 vs. G2 & $0.6(0.4-0.9)$ & \\
G2 vs. G3 & $0.8(0.6-1.1)$ & \\
\hline
\end{tabular}

Multivariate analysis of ALCAM expression, conventional markers and clinical outcome. Figures are hazard ratios with $95 \%$ confidence intervals in parentheses.

studies analyzing ALCAM expression in breast cancer $(14,19,20)$. Based on the known function of ALCAM as a cell adhesion molecule, it appears possible that loss of such a protein would result in more adverse cancer cell behavior due to a facilitated tissue infiltration, and, accordingly, a higher likelihood for developing metastases. However, several other studies came to different conclusions. For example, Piao et al (18) found high membranous ALCAM expression to be statistically correlated with a worse clinical course and high cytoplasmic staining with local recurrence. Hein et al (17) found high ALCAM expression linked to shorter overall survival compared to patients with low/moderate ALCAM scores, while ALCAM-negative tumors had an intermediate position. Burkhardt et al (15) reported that strong cytoplasmic ALCAM expression was significantly associated with earlier disease progression, while there was no correlation between membranous ALCAM expression and clinicopathological data. Ihnen et al (27) also failed to find an association of ALCAM protein or mRNA expression with histological type, grading or stage in a series of 162 breast cancers. It may be that some of these contradictory data are due to the relatively small patient cohorts analyzed, ranging from 56 to $347(14,15,17,18,21,27)$. The different methods used in those studies could be an additional source explaining these conflicting results. For example, some studies estimated levels of ALCAM using western blotting $(26,27)$, while others performed real-time PCR (14) or IHC $(15,17,27-29)$.

A variable role of ALCAM expression in patient prognosis depending on the therapies applied could also contribute to variable results between studies evaluating the prognostic role of ALCAM expression in breast cancer. A predictive role of 
ALCAM expression has indeed been suggested. Ihnen et al (27) found high ALCAM mRNA expression to be positively correlated with long overall survival in patients treated with adjuvant chemotherapy in a series of 162 patients. In contrast, patients with high ALCAM mRNA expression who did not receive chemotherapy tended to have a poorer prognosis in their study. In our patient cohort, the number of patients with complete treatment information was quite limited. The small cohort with treatment information did not enable us to show an unequivocal or strong relationship between ALCAM expression and survival. Even in the subgroup treated by chemotherapy alone, where the survival differences reached statistical significance, the absolute differences were only minimal.

Prediction of prognosis is of high relevance in breast cancer. An increasing number of patients undergo prognostic evaluation by multiparameter gene expression tests, such as Oncotype $\mathrm{DX}^{\mathrm{TM}}(30,31)$. These tests are considered to be able to reduce unnecessary adjuvant chemotherapy, Oncotype $\mathrm{DX}^{\mathrm{TM}}$, for example, by evaluating the expression levels of 21 genes and thereby generating a recurrence score of low, intermediate or high risk, which supports the decision on giving adjuvant chemotherapy or not. Data have shown that the recurrence score was low in $48 \%$ of the first 20,050 tested patients and in this group there is minimal, if any, benefit from adjuvant chemotherapy $(32,33)$. On the other hand, current knowledge indicates that approximately up to $77 \%$ of breast cancer patients may be exposed to chemotherapy toxicity and costs with little or no clinical benefit (34). Given the remaining $\sim 30 \%$ of 'unnecessary' chemotherapies, these existing tests are still far from ideal. It is highly likely that existing gene tests will soon be replaced by next generation testing. Based on our data, ALCAM expression analysis may be a component of future prognostic breast cancer tests.

Another potential relevance of ALCAM expression in breast cancer, is its membranous expression, which automatically makes ALCAM a potential therapeutic target. Wiiger et al (16) described a human recombinant single-chain antibody targeting ALCAM that was able to inhibit cancer cell invasion in vitro and in vivo tumor growth. Furthermore, Roth et al (35) described an internalizing single chain antibody that targets ALCAM in prostate cancer cells and immunoliposomes using this antibody were developed in order to deliver liposomal drugs to prostate cancer cells (35). These cell line experiments illustrate that ALCAM may be of interest not only as a classical membranous target structure for antibody therapy, but also as an internalizing epitope for intracellular delivery of liposomal drugs.

In summary, our data demonstrate that significant levels of membranous ALCAM expression occur in most breast cancers. This makes breast cancer a suitable target for antiALCAM therapies once these become available. Moreover, reduced ALCAM expression was strongly linked to adverse clinical features, making ALCAM a candidate biomarker for next generation prognostic multigene panels in breast cancer.

\section{Acknowledgements}

The authors acknowledge the technical support of Christina Koop.

\section{References}

1. Ohneda O, Ohneda K, Arai F, et al: ALCAM (CD166): its role in hematopoietic and endothelial development. Blood 98: 2134-2142, 2001

2. Masedunskas A, King JA, Tan F, et al: Activated leukocyte cell adhesion molecule is a component of the endothelial junction involved in transendothelial monocyte migration. FEBS Lett 580: 2637-2645, 2006.

3. Swart GW: Activated leukocyte cell adhesion molecule (CD166/ ALCAM): developmental and mechanistic aspects of cell clustering and cell migration. Eur J Cell Biol 81: 313-321, 2002.

4. Degen WG, van Kempen LC, Gijzen EG, et al: MEMD, a new cell adhesion molecule in metastasizing human melanoma cell lines, is identical to ALCAM (activated leukocyte cell adhesion molecule). Am J Pathol 152: 805-813, 1998.

5. van Kempen LC, van den Oord JJ, van Muijen GN, Weidle UH, Bloemers HP and Swart GW: Activated leukocyte cell adhesion molecule/CD166, a marker of tumor progression in primary malignant melanoma of the skin. Am J Pathol 156: 769-774, 2000.

6. Tomita K, van Bokhoven A, Jansen C, et al: Activated leukocyte cell adhesion molecule (ALCAM) expression is associated with a poor prognosis for bladder cancer patients. UroOncology 3 : 121-129, 2003.

7. Weichert W, Knösel T, Bellach J, Dietel M and Kristiansen G: ALCAM/CD166 is overexpressed in colorectal carcinoma and correlates with shortened patient survival. J Clin Pathol 57: 1160-1164, 2004

8. Verma A, Shukla NK, Deo SV, Gupta SD and Ralhan R: MEMD/ ALCAM: a potential marker for tumor invasion and nodal metastasis in esophageal squamous cell carcinoma. Oncology 68: 462-470, 2005.

9. Kahlert C, Weber H, Mogler C, et al: Increased expression of ALCAM/CD166 in pancreatic cancer is an independent prognostic marker for poor survival and early tumour relapse. $\mathrm{Br} \mathbf{J}$ Cancer 101: 457-464, 2009.

10. Sawhney M, Matta A, Macha MA, et al: Cytoplasmic accumulation of activated leukocyte cell adhesion molecule is a predictor of disease progression and reduced survival in oral cancer patients. Int J Cancer 124: 2098-2105, 2009.

11. Mezzanzanica D, Fabbi M, Bagnoli M, et al: Subcellular localization of activated leukocyte cell adhesion molecule is a molecular predictor of survival in ovarian carcinoma patients. Clin Cancer Res 14: 1726-1733, 2008.

12. Corrias MV, Gambini C, Gregorio A, et al: Different subcellular localization of ALCAM molecules in neuroblastoma: association with relapse. Cell Oncol 32: 77-86, 2010.

13. Tomita K, van Bokhoven A, Jansen CF, Bussemakers MJ and Schalken JA: Coordinate recruitment of E-cadherin and ALCAM to cell-cell contacts by $\alpha$-catenin. Biochem Biophys Res Commun 267: 870-874, 2000.

14. King JA, Ofori-Acquah SF, Stevens T, Al-Mehdi AB, Fodstad O and Jiang WG: Activated leukocyte cell adhesion molecule in breast cancer: prognostic indicator. Breast Cancer Res 6: R478-R487, 2004.

15. Burkhardt M, Mayordomo E, Winzer KJ, et al: Cytoplasmic overexpression of ALCAM is prognostic of disease progression in breast cancer. J Clin Pathol 59: 403-409, 2006.

16. Wiiger MT, Gehrken HB, Fodstad $\varnothing$, Maelandsmo GM and Andersson Y: A novel human recombinant single-chain antibody targeting CD166/ALCAM inhibits cancer cell invasion in vitro and in vivo tumour growth. Cancer Immunol Immunother 59: 1665-1674, 2010.

17. Hein S, Muller V, Kohler N, et al: Biologic role of activated leukocyte cell adhesion molecule overexpression in breast cancer cell lines and clinical tumor tissue. Breast Cancer Res Treat 129: 347-360, 2011.

18. Piao D, Jiang T, Liu G, Wang B, Xu J and Zhu A: Clinical implications of activated leukocyte cell adhesion molecule expression in breast cancer. Mol Biol Rep 39: 661-668, 2012.

19. Davies S and Jiang WG: ALCAM, activated leukocyte cell adhesion molecule, influences the aggressive nature of breast cancer cells, a potential connection to bone metastasis. Anticancer Res 30: 1163-1168, 2010.

20. Milde-Langosch K, Janke S, Wagner I, et al: Role of Fra-2 in breast cancer: influence on tumor cell invasion and motility. Breast Cancer Res Treat 107: 337-347, 2008. 
21. Jezierska A, Matysiak W and Motyl T: ALCAM/CD166 protects breast cancer cells against apoptosis and autophagy. Med Sci Monit 12: BR263-BR273, 2006.

22. Ruiz C, Seibt S, Al Kuraya K, et al: Tissue microarrays for comparing molecular features with proliferation activity in breast cancer. Int J Cancer 118: 2190-2194, 2006.

23. Elston CW and Ellis IO: Pathological prognostic factors in breast cancer. I. The value of histological grade in breast cancer: experience from a large study with long-term follow-up. Histopathology 19: 403-410, 1991.

24. McShane LM, Altman DG, Sauerbrei W, Taube SE, Gion M, Clark GM; Statistics Subcommittee of NCI-EORTC Working Group on Cancer Diagnostics: REporting recommendations for tumor MARKer prognostic studies (REMARK). Breast Cancer Res Treat 100: 229-235, 2006.

25. Al-Kuraya K, Schraml P, Torhorst J, et al: Prognostic relevance of gene amplifications and coamplifications in breast cancer. Cancer Res 64: 8534-8540, 2004.

26. Kilic E, Milde-Langosch K, Müller V, Wirtz R and Ihnen M Expression of activated leukocyte cell adhesion molecule in breast cancer. Predictability of the response to taxane-free chemotherapy. Pathologe 29 (Suppl 2): S347-S352, 2008 (In German).

27. Ihnen M, Müller V, Wirtz RM, et al: Predictive impact of activated leukocyte cell adhesion molecule (ALCAM/CD166) in breast cancer. Breast Cancer Res Treat 112: 419-427, 2008.
28. Ihnen M, Köhler N, Kersten JF, et al: Expression levels of activated leukocyte cell adhesion molecule (ALCAM/CD166) in primary breast carcinoma and distant breast cancer metastases. Dis Markers 28: 71-78, 2010.

29. King JA, Tan F, Mbeunkui F, et al: Mechanisms of transcriptional regulation and prognostic significance of activated leukocyte cell adhesion molecule in cancer. Mol Cancer 9: 266, 2010.

30. Paik S, Shak S, Tang G, et al: A multigene assay to predict recurrence of tamoxifen-treated, node-negative breast cancer. $\mathrm{N}$ Engl J Med 351: 2817-2826, 2004

31. Glas AM, Floore A, Delahaye LJ, et al: Converting a breast cancer microarray signature into a high-throughput diagnostic test. BMC Genomics 7: 278, 2006.

32. Paik S, Tang G, Shak S, et al: Gene expression and benefit of chemotherapy in women with node-negative, estrogen receptorpositive breast cancer. J Clin Oncol 24: 3726-3734, 2006.

33. Sparano JA and Paik S: Development of the 21-gene assay and its application in clinical practice and clinical trials. J Clin Oncol 26: 721-728, 2008

34. Carlson JJ and Roth JA: The impact of the Oncotype Dx breast cancer assay in clinical practice: a systematic review and metaanalysis. Breast Cancer Res Treat 141: 13-22, 2013.

35. Roth A, Drummond DC, Conrad F, et al: Anti-CD166 single chain antibody-mediated intracellular delivery of liposomal drugs to prostate cancer cells. Mol Cancer Ther 6: 2737-2746, 2007. 